\title{
Impact of piezocision on orthodontic tooth movement
}

\author{
Nikolaos Papadopoulos ${ }^{\mathrm{a}}$ (1) \\ Nicola Beindorff ${ }^{b}$ \\ Stefan Hoffmann ${ }^{\text {a }}$ \\ Paul-Georg \\ Jost-Brinkmann ${ }^{\mathrm{a}}$ \\ Thomas Michael Präger ${ }^{\mathrm{a}}$ (1)
}

${ }^{\mathrm{a}}$ Charité - Universitätsmedizin Berlin, Corporate Member of Freie Universität Berlin, Humboldt-Universität zu Berlin, and Berlin Institute of Health, Institute of Dental and Craniofacial Sciences, Department of Orthodontics, Dentofacial Orthopedics and

Pedodontics, Berlin, Germany

${ }^{\text {b} C h a r i t e ́ ~-~ U n i v e r s i t a ̈ t s m e d i z i n ~ B e r l i n, ~}$ Corporate Member of Freie Universität Berlin, Humboldt-Universität zu Berlin, and Berlin Institute of Health, Berlin Experimental Radionuclide Imaging Center (BERIC), Berlin, Germany
Objective: This study investigated the impact of a single piezocision in the maxillary alveolar process on the speed of tooth movement. The null hypothesis was that the speed of tooth movement will be equal with and without piezocision. Methods: All maxillary molars on one side were moved against the combined incisors in 10 ten-week-old male Wistar rats. Under general anesthesia, a force of $25 \mathrm{cN}$ was applied on either side using a Sentalloy closed coil spring. After placing the orthodontic appliance, vertical corticision was performed using a piezotome under local anesthesia, $2 \mathrm{~mm}$ mesial from the mesial root of the first molar on a randomly selected side; the other side served as the control. At the beginning of the treatment, and 2 and 4 weeks later, skull micro-computed tomography was performed. After image reconstruction, the distance between the mesial root of the first molar and the incisive canal, and the length of the mesial root of the first maxillary molar were measured. Moreover, the root resorption score was determined as described by $\mathrm{Lu}$ et al. Results: Significantly higher speed of tooth movement was observed on the corticision side; thus, the null hypothesis was rejected. The loss of root length and root resorption score were significantly more pronounced after piezocision than before. A strong correlation was observed between the speed of tooth movement and root resorption on the surgical side, but the control side only showed a weak correlation. Conclusions: Piezocision accelerates orthodontic tooth movement and causes increased root resorption.

[Korean J Orthod 2021;51(6):366-374]

Key words: Orthodontic tooth movement, Micro-computed tomography, Piezocision, Root resorption

Received February 15, 2021; Revised June 14, 2021; Accepted July 2, 2021.

Corresponding author: Thomas Michael Präger.

Private Lecturer, Charité - Universitätsmedizin Berlin, Corporate Member of Freie Universität Berlin, Humboldt-Universität zu Berlin, and Berlin Institute of Health, Institute of Dental and Craniofacial Sciences, Department of Orthodontics, Dentofacial Orthopedics and Pedodontics, Aßmannshauser Straße 4-6, 14197 Berlin, Germany. Tel +49-177-4137216 e-mail thomas.praeger@gmx.de

How to cite this article: Papadopoulos N, Beindorff N, Hoffmann S, Jost-Brinkmann PG, Präger TM. Impact of piezocision on orthodontic tooth movement. Korean J Orthod 2021;51:366374.

(C) 2021 The Korean Association of Orthodontists.

This is an Open Access article distributed under the terms of the Creative Commons Attribution Non-Commercial License (http://creativecommons.org/licenses/by-nc/4.0) which permits unrestricted non-commercial use, distribution, and reproduction in any medium, provided the original work is properly cited. 


\section{INTRODUCTION}

Surgical procedures like orthognathic surgery, distraction osteogenesis, ${ }^{1}$ and osteotomies or corticotomies of the alveolar process ${ }^{2}$ are equally effective at enhancing orthodontic tooth movement by intensifying bone remodeling. This effect is attributed to the rapid acceleratory phenomenon (RAP), which is characterized by a transient increase in bone turnover and a reduction in mineral content in the region of injury. However, the invasiveness of these procedures is often perceived as unpleasant by patients, ${ }^{4}$ and this limits their acceptance.

Recent studies indicated that less invasive surgical procedures, such as osteoperforation ${ }^{5}$ and corticision, ${ }^{6}$ are equally suitable for promoting bone remodeling in the alveolar process. Piezocision is a minimally invasive procedure in which corticision is performed using ultrasound, and it offers relative protection to the soft tissues. Piezocision has previously yielded encouraging results. $^{7}$

However, a systematic review by $\mathrm{Yi}$ et al. ${ }^{8}$ yielded only weak evidence showing that piezocision is a safe adjunct to accelerate orthodontic tooth movement, at least in the short term, and highlighted the need for more research. A study examining the impact of piezocision on orthodontic tooth movement in rats demonstrated that the speed of tooth movement in the group with piezocision was more than double that in a control group with orthodontic movement alone. ${ }^{9}$ However, these observations were based on a very small number of animals at each point of measurement, and interindividual differences might have distorted the outcomes. In addition, the covered distances in the abovementioned and several other experimental studies ${ }^{10-12}$ might be challenged by the fact that neighboring molars or the incisors, which show equal tooth movement or dislocation with growth, served as the reference to measure the covered distance. To overcome these shortcomings, a prospective study based on multiple sequential measurements in relation to a reliable fixed point and including a statistically relevant number of animals would be desirable.

Root resorption is an important adverse effect of orthodontic tooth movement, and the impact of an additional surgical procedure on it remains disputed. A clinical study reported an elevated risk of root resorption related to piezocision-supported orthodontic tooth movement. ${ }^{13}$ However, increased osteoclastic activity also enhances the bone remodeling rate and might consequently diminish the osseous resistance against the moving root, thereby potentially reducing overall root resorption.

Therefore, the aim of this study was to assess the influence of a single piezocision on the speed of orthodontic tooth movement and to determine its impact on root resorption. The null hypothesis was that there will be no difference between the amount of orthodontic tooth movement with or without piezocision.

\section{MATERIALS AND METHODS}

\section{Animal model}

The study protocol was approved by the Local Committee for Animal Care (reference number G 0189/13) according to the German Law for the Protection of Animals. All applicable institutional and national guidelines for the care and use of animals were followed.

The study included a total of 10 male Wistar rats (weight, 300-350 g; age, 10 weeks). The animals were obtained from Charles River Laboratories (Research Models and Services, Sulzfeld, Germany) and acclimatized for 1 week in the animal care facility at the Berlin Experimental Radionuclide Imaging Center, Charité - Universitätsmedizin Berlin, on a 12:12-h light/dark cycle, under a constant temperature of $22^{\circ} \mathrm{C}$ and humidity of $50 \%$. The rats had free access to rat chow pellets and water (Chow Pellets, Fa. Ssniff, Soest, Germany). Additionally, soft foods like cottage cheese and softened rusk were fed. The changes in weight were monitored; accordingly, the baseline weight was measured on day 0 for all animals before the procedures were performed and on a daily basis thereafter until the end of the experiment.

\section{Orthodontic appliance}

The orthodontic appliance was placed into the maxilla according to an established protocol. ${ }^{14}$ However, it was slightly modified as shown in Figure 1. General anesthesia was induced via intraperitoneal administration of medetomidine (Domitor, $0.135 \mathrm{mg} / \mathrm{kg}$ body weight) and ketamine (Ketalar, $0.25 \mathrm{mg} / \mathrm{kg}$ body weight). Under local anesthesia (UDS forte; Sanofi-Aventis, Frankfurt/M., Germany), shallow notches were made in the enamel of the molars (buccal and palatal surfaces) and both incisors (circumferentially) above the gingival margin by using a handpiece equipped with a small round bur in order to enhance the stability of ligation.

All molars on one side and both incisors were then interconnected using an orthodontic wire ligature (0.08" Drahtligaturen; SmileDental, Düsseldorf, Germany) that was recessed into the grooves and embedded in composite resin via the acid-etch technique (37\% phosphoric acid gel, Monobond Plus and Tetric Flow; Ivoclar Vivadent, Schaan, Liechtenstein) and light curing. Subsequently, a 25-cN Sentalloy Closed Coil Spring Ultra Light (Sentalloy, item 10-000-26, 25 g; GAC International, Gräfelfing, Germany) was placed between the three molars on each side and the connected incisors. At the end of the procedure, all animals received intramuscular 


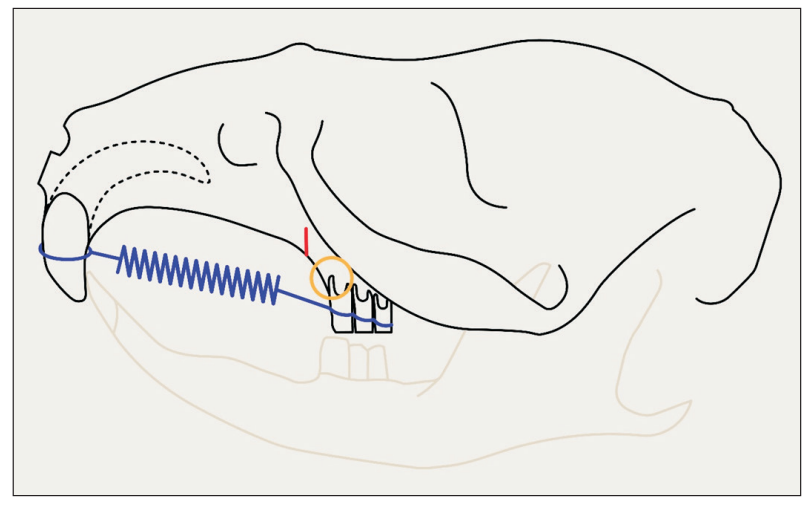

Figure 1. Orthodontic appliance consisting of wire ligatures embedded in composite resin and a coil spring placed between the three molars of one side and both the incisors. The piezocision cut is depicted in red, and is placed $2 \mathrm{~mm}$ mesial from the mesial root of the first molar. The area inside the circle is investigated for root resorption.

injections of flunixine (Finadyne, $1 \mathrm{mg} / \mathrm{kg}$ body weight) twice a day as an analgesic.

\section{Surgical procedure}

The surgical side was predetermined in every animal by using a randomized list generated by drawing lots prior to the beginning of the laboratory experiments. Following the insertion of the orthodontic appliance, additional local anesthesia was administered mesial from the first molar on the selected side. Subsequently, a vertical incision of the buccal alveolar soft tissue, ranging from the alveolar ridge to the level of the root tips, was made using a microsurgical scalpel and blade (BB364R; Aesculap, Tuttlingen, Germany). The exposed cortical bone was then cut in a vertical direction by using a piezotome equipped with the BS5 tip (Piezotome 2; Acteon Satelec, Mérignac, France), which was adjusted to deliver low-frequency ultrasonic waves $(28-36 \mathrm{kHz})$ under continuous irrigation of isotonic saline solution. Finally, the wound margins were readapted.

\section{Imaging}

Before (T0) the placement of the orthodontic appliance and at 2 weeks (T1) and 4 weeks (T2) after appliance placement, micro-computed tomography $(\mu \mathrm{CT})$ scans (NanoSPECT/CTplus scanner; Mediso Medical Imaging Systems, Budapest, Hungary, Figure 2) of the skull were performed under general inhalation anesthesia (1.3-2.0\% isoflurane with oxygen). The scans lasted 15 minutes, and the acquisition parameters were $55 \mathrm{kV}$, $155 \mathrm{~mA}, 1,500-\mathrm{ms}$ exposure time, and $360^{\circ}$ rotation. The reconstructed cubic voxel side size was $96 \mu \mathrm{m}$ in a $198 \times 198 \times 546$ pixel matrix. Following the third imag-

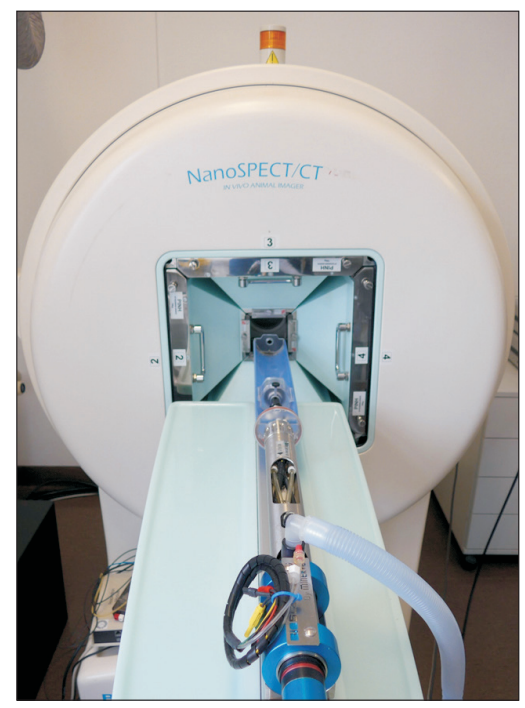

Figure 2. The NanoSPECT/CTplus scanner (Mediso Medical Imaging Systems, Budapest, Hungary) used for in vivo imaging.

ing at $\mathrm{T} 2$, the animals were euthanized by administering an intraperitoneal overdose of the barbiturate thiopental sodium.

\section{Measurements}

All steps related to the analysis of the $\mu \mathrm{CT}$ data were performed by one orthodontist (N.P.) with long-term experience in $\mu \mathrm{CT}$ evaluation using the PMOD software Version 3.4 (PMOD Technologies Ltd, Zurich, Switzerland) at a magnification of $\times 200$. All images were reconstructed from the datasets obtained from the $\mu \mathrm{CT}$ scanner and consequently stored as DICOM (Digital lmaging and Communications in Medicine) files.

\section{Orthodontic tooth movement}

Orthodontic tooth movement was investigated by measuring the distances between the distal border of the incisive canal and the apical tip of the mesial root of the first molar on each side (Figure 3). The differences between the distances at T0 and T1 represented the tooth movement during the first 2 weeks; those between T1 and T2 represented that during the second 2 weeks; and the overall tooth movement was calculated as T0 minus T2. As the incisive canal was stable during craniofacial development in the rat, ${ }^{15}$ we used it as our anterior reference structure. Three-dimensional (3D) images of the maxilla were reconstructed and reproducibly positioned such that the palate was parallel to the horizontal plane and the coronal plane was rectangular to the horizontal plane through the center of both the condylar heads. The third plane was reconstructed perpendicular to both the other planes through the incisive canal. These planes 


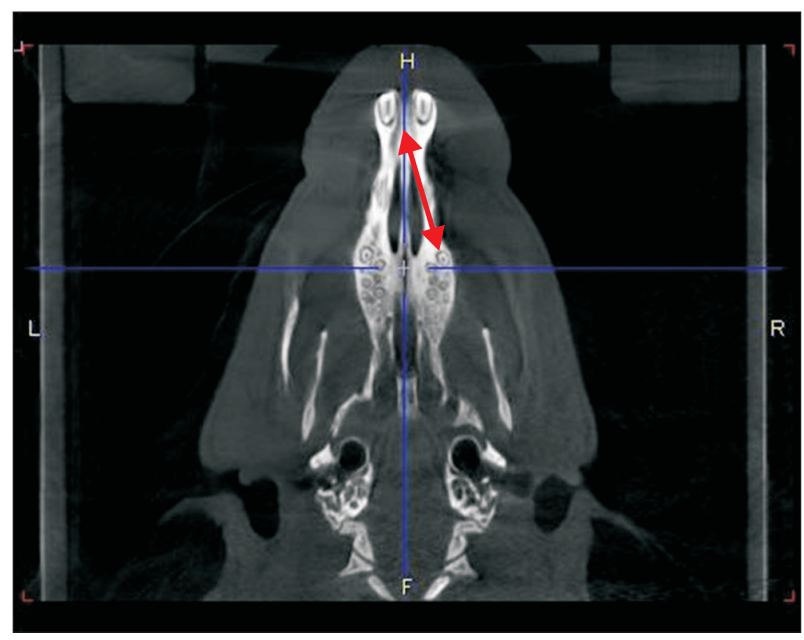

Figure 3. Reconstructed micro-computed tomography image for measuring the distance between the incisive canal and the apex of the mesial root of the first molar (red arrow).

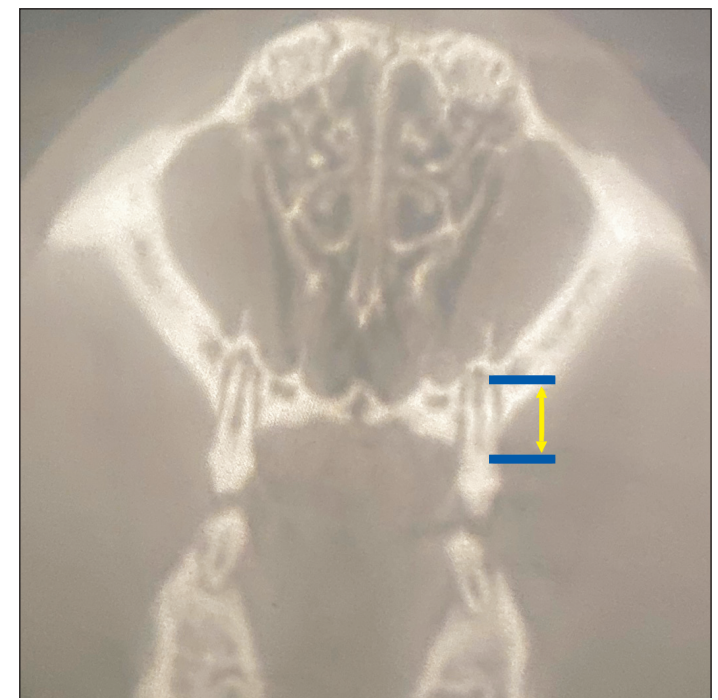

Figure 4. Measuring the root length of the mesial root of the first molar from the trifurcation to the apex in the axial plane (yellow arrow).

allowed for reproducible positioning of all maxillae in a comparable position.

\section{Root resorption}

The 3D images were reconstructed such that the axis of the mesial root of each first molar (Figure 4) was perpendicular to the horizontal plane. The length of the mesial root of the first molar was defined as the distance between the trifurcation and the apical tip. For measuring the length of the root, we considered the tri-

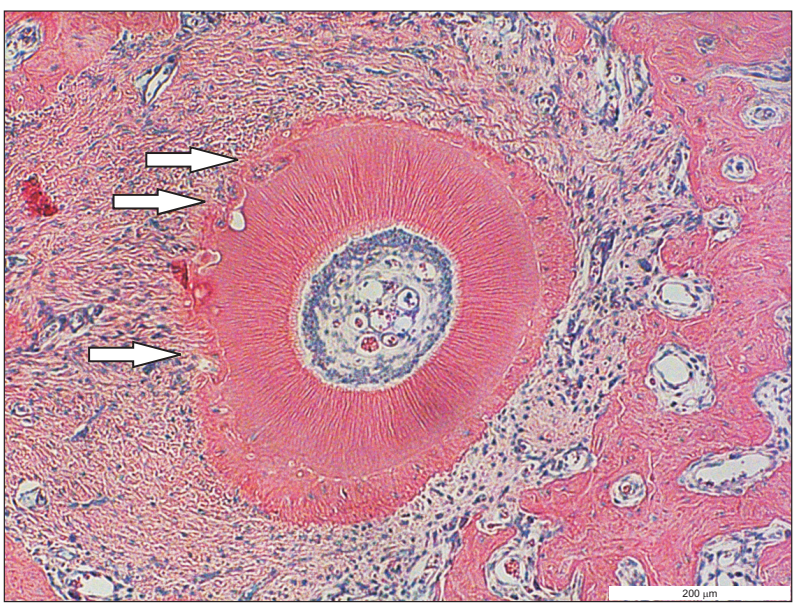

Figure 5. Hematoxylin-eosin-stained specimen of the mesial root of the first maxillary molar. The arrows display areas with root resorption on the mesial side of the root.

furcation to be stable. Owing to artifacts caused by the orthodontic appliance, we could not reliably measure the root length at T1. Consequently, the data for root resorption were limited to those obtained at T0 and $\mathrm{T} 2$.

\section{Histology}

\section{Samples for histological examination}

At the end of the experiment, all animals were euthanized via anesthetic overdosage. All maxillae were cautiously prepared, retrieved, and placed for 48 hours in 4\% paraformaldehyde (Sigma-Aldrich, St. Louis, MO, USA) in phosphate-buffered saline. Following decalcification with 15\% dipotassium ethylenediaminetetraacetic acid over 12 weeks, the samples were dehydrated using graded alcohol washes and then embedded in paraffin with their bases parallel to the palatal plane. Subsequently, $5-\mu \mathrm{m}$-thick sections were cut horizontally by using a conventional microtome, and the sections were then stained using hematoxylin-eosin.

All sections were assessed qualitatively and quantitatively under an Olympus $\mathrm{BH} 2$ Microscope (Olympus Optical Co. Ltd, Tokyo, Japan). The periradicular region of the mesial root of the first molar was investigated for root resorption lacunae. Additionally, the healing of the piezocision wound was compared to that of the homologous region on the other side.

\section{Root resorption score}

To assess and quantify the extent of root resorption, the root resorption score was determined on magnified pictures (magnification $\times 120$ ) in the root region on the basis of the methodology suggested by Lu et al. ${ }^{16}$ The root resorption score was calculated as the percent- 
age of grids in the radicular area with root resorption divided by the total number of investigated grids in the radicular areas (Figure 5).

\section{Statistics}

The power analysis was conducted using G*Power software for Windows, Version 3.1.9.2 (Heinrich-HeineUniversität, Düsseldorf, Germany), with an $\alpha$ level of 0.05 and a $\beta$ level of 0.8 on the basis of data from a previously published study ${ }^{14}$ and with a Cohen's $d$ of 1.2. The analysis showed that a minimum number of 8 animals were required per group. All data sets were separately tested for normal distribution by using the Shapiro-Wilk test and for homoscedasticity by using the Levene test. Consequently, group differences for tooth movement were assessed using the paired two-sided Student's ttest and those for root lengths, whose data were not normally distributed, were assessed using the Wilcoxon signed-rank test. A potential relationship between both datasets was investigated by using Spearman's rank correlation coefficient $\rho$. The level of significance was set at 0.05 . Descriptive statistics included mean, standard deviation, minimum, and maximum. All data were processed with Excel (Microsoft, Redmond, WA, USA) and PASW Statistics for Windows, Version 18.0 (SPSS Inc., Chicago, IL, USA).

\section{Method error}

To determine the method error, the distances for orthodontic tooth movement and for root resorption were measured independently on 10 randomly selected $\mu \mathrm{CT}$ images acquired more than 4 weeks after the first measurement. The method error according to Dahlberg was calculated using the following formula:

$$
M E=\sqrt{\sum_{i=1}^{n} \frac{d_{i}^{2}}{2 n}},
$$

where $d_{i}$ was the difference between the two repeated measurements, $\mathrm{n}$ was the sample size, and ME was the method error. ${ }^{17}$ Student's $t$-tests were used to verify whether the differences between the first and second measurements were significant.

\section{RESULTS}

\section{Animals}

All 10 rats included in this study remained healthy and showed a continuous increase in body weight until the endpoint.

\section{Tooth movement}

All data on tooth movement are displayed in Tables 1 and 2.

\section{Orthodontics only side}

The initial distance between the tip of the mesial root of the first molar and the incisive canal was $10.97 \pm$ $0.26 \mathrm{~mm}$ (mean \pm standard deviation [SD]) before tooth movement, and it decreased to $10.13 \pm 0.23 \mathrm{~mm}$ at the end of treatment. We observed an average movement of $0.45 \pm 0.29 \mathrm{~mm}$ during the first 2 weeks. The speed of movement slightly decreased to $0.39 \pm 0.21 \mathrm{~mm}$ in the second 2 weeks, thereby resulting in an overall movement of $0.84 \pm 0.24 \mathrm{~mm}$ after 4 weeks.

\section{Surgical side}

The distance between the tip of the mesial root of the first molar and the incisive canal decreased from $10.97 \pm$ $0.24 \mathrm{~mm}$ (mean $\pm \mathrm{SD}$ ) before tooth movement to $9.82 \pm$ $0.24 \mathrm{~mm}$ afterwards. After piezocision, an average movement of $0.53 \pm 0.29 \mathrm{~mm}$ occurred during T0-T1, and an average movement of $0.62 \pm 0.26 \mathrm{~mm}$ occurred during $\mathrm{T} 1-\mathrm{T} 2$. The overall movement was $1.15 \pm 0.27 \mathrm{~mm}$.

\section{Comparison}

The movements on the surgical side were larger than those on the orthodontics only side, but this difference was significant only during the second half $(p<0.05)$ and highly significant $(p<0.0005)$ only when considering the overall tooth movement. The overall speed of tooth movement was $0.84 \mathrm{~mm} / 4$ weeks and $1.15 \mathrm{~mm} / 4$ weeks, respectively. The increase in tooth movement was

Table 1. Distances between the apex of the mesial root of the first molar and the incisive canal in millimeters

\begin{tabular}{|c|c|c|c|c|c|c|}
\hline \multirow[b]{2}{*}{ Statistical value } & \multicolumn{3}{|c|}{ Orthodontics only side } & \multicolumn{3}{|c|}{ Surgical side } \\
\hline & $\begin{array}{c}\text { Distance } \\
\text { T0 }\end{array}$ & $\begin{array}{c}\text { Distance } \\
\text { T1 }\end{array}$ & $\begin{array}{c}\text { Distance } \\
\text { T2 }\end{array}$ & $\begin{array}{c}\text { Distance } \\
\text { T0 }\end{array}$ & $\begin{array}{c}\text { Distance } \\
\text { T1 }\end{array}$ & $\begin{array}{c}\text { Distance } \\
\text { T2 }\end{array}$ \\
\hline Mean & 10.97 & 10.52 & 10.13 & 10.97 & 10.44 & 9.82 \\
\hline Standard deviation & 0.26 & 0.22 & 0.23 & 0.24 & 0.26 & 0.24 \\
\hline Maximum & 11.32 & 11.03 & 10.58 & 11.32 & 11.03 & 10.14 \\
\hline Minimum & 10.44 & 10.14 & 9.85 & 10.58 & 10.14 & 9.41 \\
\hline
\end{tabular}

Distances before orthodontic treatment (T0), after 2 weeks (T1), and after 4 weeks (T2), measured in 10 animals. 
Papadopoulos et al • Piezocision and orthodontic tooth movement

Table 2. Tooth movement during the first two weeks (T0-T1), the second two weeks (T1-T2), and the entire period (T0-T2) in millimeters

\begin{tabular}{ccccc}
\hline Period & Orthodontics only side $(\mathbf{n}=\mathbf{1 0}$ rats $)$ & Surgical side $(\mathbf{n}=\mathbf{1 0}$ rats $)$ & \multicolumn{1}{c}{$\boldsymbol{\Delta}$ in $\%$} & $\boldsymbol{p}$-value \\
\hline T0-T1 & $0.45 \pm 0.29(0.00,0.85)$ & $0.53 \pm 0.29(0.00,0.88)$ & 0.127 & 59 \\
T1-T2 & $0.39 \pm 0.21(0.14,0.74)$ & $0.62 \pm 0.26(0.15,1.18)$ & $0.011^{*}$ \\
T0-T2 & $0.84 \pm 0.24(0.29,1.18)$ & $1.15 \pm 0.27(0.59,1.47)$ & 37 & $0.000^{* * *}$ \\
\hline
\end{tabular}

Values are presented as mean \pm standard deviation (minimum, maximum).

$\Delta$ equals the increase produced by piezocision.

T0, initial; T1, 2 weeks later; T2, 4 weeks later.

Differences between two sides were tested by paired $t$-tests and considered significant at $p<0.05 .{ }^{*} p<0.05 ;{ }^{* * *} p<0.001$.

Table 3. Root lengths before (T0) and after orthodontic treatment (T2) in millimeters

\begin{tabular}{ccccc}
\hline Timing & Orthodontics only side (n= 10 rats) & Surgical side (n= 10 rats) & p-value & NA \\
T0 & $2.59 \pm 0.24(2.06,2.94)$ & $2.60 \pm 0.19(2.21,2.79)$ & NA \\
T2 & $2.47 \pm 0.23(1.91,2.79)$ & $2.32 \pm 0.17(2.06,2.50)$ & $0.041^{*}$ \\
T2-T0 & $-0.12 \pm 0.09(-0.29,0.00)$ & $-0.28 \pm 0.12(-0.44,-0.15)$ & \\
\hline
\end{tabular}

Values are presented as mean \pm standard deviation (minimum, maximum).

T2-T0 indicates amount of root resorption.

NA, not available.

Differences between two sides were tested by paired $t$-tests and considered significant at $p<0.05 .{ }^{*} p<0.05$.

Table 4. Root resorption score with and without piezocision

\begin{tabular}{ccc}
\hline $\begin{array}{c}\text { Orthodontics only } \\
\text { side }\end{array}$ & $\begin{array}{c}\text { Surgical } \\
\text { side }\end{array}$ & p-value \\
\hline $23.0 \pm 4.4(16,29)$ & $29.6 \pm 4.5(23,37)$ & $<0.005$ \\
\hline
\end{tabular}

Values are presented as mean \pm standard deviation (minimum, maximum).

$18 \%$ in the first 2 weeks and $59 \%$ in weeks 3 and 4 . The overall difference between orthodontics only and piezocision plus orthodontics was 37\%.

\section{Root resorption}

Data on root resorption are shown in Table 3.

\section{Orthodontics only side}

Root resorption was detectable in all but one animal and amounted to an average $( \pm \mathrm{SD})$ of $0.12 \pm 0.09 \mathrm{~mm}$.

\section{Surgical side}

On the surgical side, root resorption after piezocision was observed in all animals. The resorption amounted to an average $( \pm$ SD) of $0.28 \pm 0.12 \mathrm{~mm}$.

\section{Comparison}

Root resorption was an almost ubiquitous phenomenon, but it was more prominent after surgery. This difference was significant $(p<0.05)$.
Table 5. The correlation between the overall tooth movement and root resorption

\begin{tabular}{lcc}
\hline Statistical value & Orthodontics only & Piezocision \\
\hline Spearman's $\rho$ & 0.63 & 0.97 \\
$p$-value & 0.053 & 0.001 \\
\hline
\end{tabular}

Spearman's rank correlation test was performed. Spearmans' rho indicates the correlation was weak and not significant for the orthodontics only side, but strong and significant after piezocision.

\section{Root resorption score}

Data on the root resorption score are shown in Table 4.

\section{Orthodontics only side}

Histological examination revealed resorption lacunae in every animal that received orthodontic treatment, thus resulting in a root resorption score of $23.0 \pm 4.4$ without additional piezocision.

\section{Surgical side}

On the surgical side, the histological images were qualitatively comparable and the presence of resorption lacunae was more frequent than on the orthodontics only side $(29.6 \pm 4.5)$.

\section{Comparison}

Root resorption was more frequent in animals treated using piezocision. This difference was significant $(p<$ 0.005). 


\section{Relationship between tooth movement and root resorption}

A weak correlation was observed between tooth movement and root resorption in the orthodontics only group, but a strong correlation was observed when piezocision preceded orthodontic tooth movement (Table 5). On determining the method error, no significant difference was found between the first and second measurements.

\section{DISCUSSION}

To our best knowledge, this is the first animal study to investigate the speed of orthodontic tooth movement following piezocision by performing sequential measurements in the same animals. Dibart et al..$^{9}$ demonstrated an acceleration of tooth movement resulting from piezocision cuts; however, their observations were based on pooled data from only three to five animals per time point, which did not allow for extended statistical analysis.

Following a power analysis, which specified the required minimum number of animals as 10 per group, and influenced by animal welfare guidelines, we decided on a split-mouth design that provided the additional advantage of reducing possible distortions caused by interindividual variations. To improve the reliability of our measurements, we selected a stable anatomical structure as the reference point instead of mobile structures like the incisors or another molar. In growth studies, implants served as fixed points, ${ }^{18}$ but this would have necessitated inflicting an additional surgical trauma to the animals. Certain anatomical structures like the incisive canal are considered stable at this stage of craniofacial development and could therefore function as a reference point. ${ }^{15}$ Consequently, the distance covered by the root tip of the first molar should approach the true tooth movement over time without any distortion caused by a possible movement of the reference point.

An important purpose of our study was to approach the limits of surgically supported orthodontics and to determine the minimal dose of osseous trauma needed to promote orthodontic tooth movement. Acceleration after corticotomies has been well demonstrated, and multiple piezocision cuts have been known to enhance orthodontic tooth movement. ${ }^{9,11}$ However, under clinical conditions and considering that the majority of our orthodontic patients are children and youth, a procedure with reduced invasiveness is desirable. Therefore, we limited the surgical intervention to a single piezocision cut that was performed at a safe distance to the dental roots in order to limit the risk of iatrogenic root damage. This risk was highlighted by Patterson et al. ${ }^{13}$ who reported that more than one-third of the patients expe- rienced iatrogenic root damage after piezocision cuts.

The greater part of the accelerated tooth movement took place during the third and fourth weeks, whereas in the first 2 weeks, only a slight and statistically insignificant increase was observed. This finding is backed by comparable observations with piezocision in the rat, ${ }^{9}$ but remains contradictory to those of many other studies based on different surgical methods. ${ }^{19}$

Tooth movement is initiated by the removal of hyalinized and osseous tissue-a task that is accomplished by osteoclasts. Consequently, osteoclastogenesis is considered the decisive factor for the initiation and progression of tooth movement.

The course of orthodontic tooth movement can be subdivided into different phases. Burstone ${ }^{20}$ termed the first phase after force initiation "the lag phase," a period in which the number and function of osteoclasts is upregulated under the conditions of orthodontic tooth movement without surgery. Our findings are comparable, and the delayed acceleration we observed is well supported by the increase in osteoclastogenesis, which peaked 7 days after piezocision. ${ }^{9}$ Immediately after force application, the number of active osteoclasts, which have to mature to gain function and to eliminate the hard tissue before tooth movement, increases. Therefore, it is plausible that the second half of our experiment showed an acceleration of tooth movement based on the increase in osteoclastic function.

Another explanation for the incongruent patterns of acceleration could be the difference in orthodontic appliances used. The orthodontic appliance used in our study aligned all three molars and aimed to simulate an en masse movement. Therefore, the force application point was situated closer to the center of resistance, and this resulted in a more bodily movement compared to that produced by appliances in which a coil spring tips the first molar. A tipping movement is initiated faster because it meets less resistance in the beginning.

The piezocision cut in our study was placed distant from the second molar and even farther from the third molar. Hence, the RAP, which is by definition a centrifugal phenomenon, should have weakened with increasing distance.

Root resorption was present in both the groups, and $\mu \mathrm{CT}$ as well as histological examination provided evidence for this phenomenon. This phenomenon is observed in the majority of young orthodontic patients and is commonly related to orthodontic tooth movement. ${ }^{21,22}$ Our findings confirmed that resorption processes become detectable at the surface of the dental root, both with and without surgical intervention. However, apical and lateral root resorptions were significantly more extended in the piezocision group. As root resorption is associated with high osteoclastic activity, which is an 
equally decisive factor in the bone remodeling process, ${ }^{23}$ the risk of damaging the dental roots during piezocision has to be cautiously traded off against the advantage of a slightly accelerated orthodontic treatment.

\section{CONCLUSION}

A significant overall acceleration of orthodontic tooth movement occurred after a single piezocision cut in the area of the first molar over a period of 4 weeks. The accelerative effect was more pronounced in the second half of this period. Root resorption was detectable both radiologically and histologically at the mesial root of the first molars in both the groups, but was increased significantly with piezocision. The correlation between the overall speed of tooth movement and the amount of root resorption was strong with piezocision, but only weak without surgery.

\section{CONFLICTS OF INTEREST}

No potential conflict of interest relevant to this article was reported.

\section{ACKNOWLEDGEMENTS}

This study was supported by funds from the Orthodontic Department.

NP received a research scholarship from Forschungsgemeinschaft Dental e. V., Cologne, Germany.

\section{REFERENCES}

1. Liou EJ, Huang CS. Rapid canine retraction through distraction of the periodontal ligament. Am J Orthod Dentofacial Orthop 1998;114:372-82.

2. Wilcko W, Wilcko MT. Accelerating tooth movement: the case for corticotomy-induced orthodontics. Am J Orthod Dentofacial Orthop 2013;144:4-12.

3. Frost HM. The regional acceleratory phenomenon: a review. Henry Ford Hosp Med J 1983;31:3-9.

4. Wilcko MT, Wilcko WM, Pulver JJ, Bissada NF, Bouquot JE. Accelerated osteogenic orthodontics technique: a 1-stage surgically facilitated rapid orthodontic technique with alveolar augmentation. J Oral Maxillofac Surg 2009;67:2149-59.

5. Alikhani M, Raptis M, Zoldan B, Sangsuwon C, Lee YB, Alyami B, et al. Effect of micro-osteoperforations on the rate of tooth movement. Am J Orthod Dentofacial Orthop 2013;144:639-48.

6. Kim SJ, Park YG, Kang SG. Effects of Corticision on paradental remodeling in orthodontic tooth movement. Angle Orthod 2009;79:284-91.

7. Dibart S, Sebaoun JD, Surmenian J. Piezocision: a minimally invasive, periodontally accelerated orthodontic tooth movement procedure. Compend Contin Educ Dent 2009;30:342-4, 346, 348-50.

8. Yi J, Xiao J, Li Y, Li X, Zhao Z. Efficacy of piezocision on accelerating orthodontic tooth movement: a systematic review. Angle Orthod 2017;87:491-8.

9. Dibart S, Yee C, Surmenian J, Sebaoun JD, Baloul S, Goguet-Surmenian E, et al. Tissue response during Piezocision-assisted tooth movement: a histological study in rats. Eur J Orthod 2014;36:457-64.

10. Librizzi Z, Kalajzic Z, Camacho D, Yadav S, Nanda R, Uribe F. Comparison of the effects of three surgical techniques on the rate of orthodontic tooth movement in a rat model. Angle Orthod 2017;87:717-24.

11. Kraiwattanapong K, Samruajbenjakun B. Effects of different force magnitudes on corticotomy-assisted orthodontic tooth movement in rats. Angle Orthod 2018;88:632-7.

12. Peron AP, Johann AC, Papalexiou V, Tanaka OM, Guariza-Filho 0, Ignácio SA, et al. Tissue responses resulting from tooth movement surgically assisted by corticotomy and corticision in rats. Angle Orthod 2017;87:118-24.

13. Patterson BM, Dalci O, Papadopoulou AK, Madukuri $\mathrm{S}$, Mahon J, Petocz P, et al. Effect of piezocision on root resorption associated with orthodontic force: a microcomputed tomography study. Am J Orthod Dentofacial Orthop 2017;151:53-62.

14. Präger TM, Meyer P, Radlanski R, Jost-Brinkmann PG, Müller-Hartwich R. Microdamage in the alveolar process of rat maxillae after orthodontic tooth movement. J Orofac Orthop 2015;76:41-50.

15. Ulgen M, Baran S, Kaya H, Karadede 1. The influence of the masticatory hypofunction on the craniofacial growth and development in rats. Am J Orthod Dentofacial Orthop 1997;111:189-98.

16. Lu LH, Lee K, Imoto S, Kyomen S, Tanne K. Histological and histochemical quantification of root resorption incident to the application of intrusive force to rat molars. Eur J Orthod 1999;21:57-63.

17. Dahlberg G. Statistical methods for medical and biological students. London: Allen \& Unwin Ltd.; 1940 p. 122-32.

18. Björk A. The use of metallic implants in the study of facial growth in children: method and application. Am J Phys Anthropol 1968;29:243-54.

19. Dutra EH, Ahmida A, Lima A, Schneider S, Nanda R, Yadav S. The effects of alveolar decortications on orthodontic tooth movement and bone remodelling in rats. Eur J Orthod 2018;40:423-9.

20. Burstone CJ. The biomechanics of tooth movement. In: Kraus BS, Riedel RA, eds. Vistas in orthodontics. Philadelphia: Lea \& Febiger; 1962. p. 197-213.

21. Linge L, Linge B0. Patient characteristics and treat- 
ment variables associated with apical root resorption during orthodontic treatment. Am J Orthod Dentofacial Orthop 1991;99:35-43.

22. Kurol J, Owman-Moll P, Lundgren D. Time-related root resorption after application of a controlled continuous orthodontic force. Am J Orthod Dento- facial Orthop 1996;110:303-10.

23. lino S, Sakoda S, Ito G, Nishimori T, Ikeda T, Miyawaki S. Acceleration of orthodontic tooth movement by alveolar corticotomy in the dog. Am J Orthod Dentofacial Orthop 2007;131:448.e1-8. 